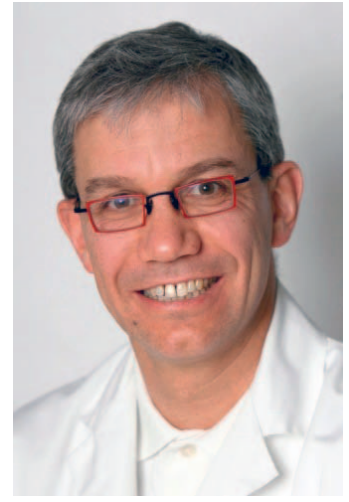

Prof. Dr. med. dent. Christian E. Besimo, Brunnen

\section{Zahnmedizin quo vadis?}

Mehr ärztliches Sehen, Denken und Handeln gefordert

Es mag Aussenstehende erstaunen, dass in einer ärztlichen Fachzeitschrift wie der ScHWEIZERISCHEN ZeITschrift FÜR GANZHEITSMEDIZIN immer wieder zahnmedizinische Themen angesprochen werden und im Editorial Board dieses Fachorgans auch Zahnärzte vertreten sind. Dies entspricht auf den ersten Blick nicht dem in den Medien und durch die zahnärztliche Praxis vermittelten Bild einer sich mehr und mehr auf sich selbst beschränkenden Zahnmedizin. Dabei wird jedoch übersehen, dass das rasch wachsende Grundlagenwissen und die zunehmende Zahl fächerübergreifender Erkenntnisse $\mathrm{zu}$ einer Vielzahl möglicher Wechselwirkungen zwischen oralen und systemischen Erkrankungen längst ein völlig anderes Bild der Zahnmedizin zeichnen $[1,2]$. Dieses fordert nachdrücklich ein immer umfassenderes Verständnis des Kauorgans als Teil des Gesamtorganismus und somit nachdrücklich die Rückbesinnung auf ärztliches Denken und Handeln sowie die dringende Notwendigkeit einer engen interdisziplinären Vernetzung mit anderen medizinischen Fachrichtungen - und nicht die selbstverliebte Beschränkung auf technische Errungenschaften bzw. kosmetische Nebenschauplätze $[3,4]$.

\section{Zeit für eine klare Entscheidung}

Soll die Zahnmedizin weiter auf der Welle des Jugendlichkeitswahns unserer Gesellschaft mitschwimmen und, entgegen ethischen Grundwerten ärztlichen Handelns, medial entfachte Defizitgefühle munter weiter schüren und auch wirtschaftlich ausnutzen [5]? Oder ist sie nicht deutlich besser beraten, sich im Sinne einer Oralen Medizin und gestützt auf das zur Verfügung stehende Grundlagenwissen auf ihren ärztlichen Auftrag der Gesunderhaltung des Kauorgans als eng vernetzter Teil des Gesamtorganismus zurückzubesinnen?

\section{Ethik der interdisziplinären Entscheidungsfindung}

Aus besagter medizinisch-ganzheitlicher Sicht stellt die zunehmende Komplexität und interdisziplinäre Vernetzung von Diagnostik und Therapie die in der Zukunft als Orale Medizin zu verstehende Zahnmedizin vor grosse fachliche und ethische Herausforderungen [6, 7]. So bleibt interdisziplinär zu definieren, welche diagnostischen Daten bei welchen gesundheitlichen Problemstellungen mit welchen Mitteln und durch wen zu erheben sind. Darauf basierend gilt es festzulegen, welche Entscheidungsprozesse erforderlich sind, um schliesslich eine adäquate Therapiewahl sicherstellen zu können, die die ethischen Grundprinzipien ärztlichen Handelns sowie gesetzliche Vorlagen zu erfüllen und in der Folge die Menschenwürde jedes einzelnen Patienten zu respektieren vermag.

Zahnärzte und Ärzte sind gefordert, ihre persönliche Erfahrung mit der Evidenz wissenschaftlicher Erkenntnisse zur Deckung und mit dem Wissensstand der anderen medizinischen Fachrichtungen in Einklang zu bringen. Entscheidungsprozesse und -kompetenzen müssen nicht nur festgelegt, sondern laufend auch überprüft und bei Bedarf angepasst werden. Eine besondere Rolle kommt dabei dem Konsens unterschiedlicher Fachgebiete bei interdisziplinär vernetzten Entscheidungsprozessen zu, will man die Qualität ethischen und ärztlichen Handelns nicht gefährden.

\section{Dialogisch-partizipativer Entscheidungsweg}

Schliesslich sind die gewählten Entscheidungsprozesse auch transparent zu dokumentieren und kommunizieren. Nicht nur die notwendigen Fachpersonen sind in die Entscheidungsprozesse einzubinden, sondern vor allem auch die Patienten und ihr soziales Umfeld. Dabei ist der ArztPatienten-Beziehung besondere Sorge zu tragen. Es muss

\section{KARGER}

Fax +497614520714 Information@Karger.d www.karger.com
Prof. Dr. med. dent. Christian E. Besimo

Leitender Arzt Zahnmedizin/Stellvertretender Chefarzt

Aeskulap-Klinik - Ärztliche Ganzheitsmedizin

Gersauerstrasse 8, 6440 Brunnen, Schweiz

Tel. +41 41 825-4922, Fax - 4863

christian.besimo@aeskulap.com 
in jedem Fall sichergestellt sein, dass unsere Patienten einerseits ihre gesundheitliche Situation verstehen, um Entscheidungen mittragen zu können, andererseits aber auch in Bezug auf Bedeutung und Auswirkung von Diagnosen bzw. Therapien durch unsere Fürsorge getragen und in der Entscheidungsfindung nicht allein gelassen werden.

\section{Mehrdimensionale Diagnostik, multidirektionale Therapie}

Grundsätzliche Voraussetzung für Ganzheitlichkeit in der Oralen Medizin ist die enge interdisziplinäre Vernetzung mit der Medizin. Gerade das Erkennen möglicher Zusammenhänge zwischen oralen und systemischen Erkrankungen bedingt diese Zusammenarbeit, ohne die die bisher übliche eindimensionale Diagnostik zum Scheitern verurteilt sein muss und keine fruchtbare Basis für eine notwendige, multidirektionale Therapie zu bieten vermag.

Oralmedizinische Diagnostik und Therapie sollten zudem in mehrere Phasen gegliedert und gerade bei systemischen Problemstellungen auf ärztliche Massnahmen abgestimmt werden. So kann die Wirkung jeder Intervention interdisziplinär evaluiert werden, bevor die Planung der nächsten Diagnose- oder Therapiephase erfolgt. Dies erlaubt nicht nur die Überprüfung der Diagnosen, sondern auch der ins Auge gefassten weiteren Behandlungsschritte auf ihre Richtigkeit. Diese Nutzung der individuellen biologischen Antwort der Patienten ist das zweite grundlegende und wissenschaftlich fundierte Prinzip der Ganzheitlichkeit, das durch keine nicht oder nur ungenügend geprüfte Diagnose- oder Therapiemethode ersetzt werden kann. Eine solche, auf Evidenz basierende Orale Medizin bietet unseren Patienten nicht nur Ganzheitlichkeit, sondern auch Qualität und Sicherheit.

\section{Literatur}

1 Besimo CE: Orale und systemische Erkrankungen. Teil 1: Eine auf Evidenz basierende Übersicht ihrer Wechselwirkungen. Schweiz Zschr Ganzheitsmedizin 2008;20:406-411.

2 Besimo CE: Orale und systemische Erkrankungen. Teil 2: Interdisziplinäres Assessment des alternden Menschen. Schweiz Zschr Ganzheitsmedizin 2009;21:54-58.

3 Besimo CE: Mehr ärztliches Sehen, Denken und Handeln sind gefordert. Zahnarzt Praxis 2009;2-3:1.

4 Palla S: Zahnmedizin am Scheideweg. Schweiz Monatsschr Zahnmed 2010;120:362-363.

5 Maio G: Zahnmedizin zwischen Heilkunde und Beauty-Industrie. Zu den ethischen Unzulänglichkeiten des Ästhetik-Booms in der Zahnheilkunde. Schweiz Monatsschr Zahnmed 2009;119:47-51.

6 Rüegger H, Hillewerth K, Schmid W: Wie werden in der Medizin gute Entscheidungen getroffen? Medizin-ethische Diskussion, Teil 1. Hausarzt Praxis 2009;2:30-32.

7 Rüegger H, Hillewerth K, Schmid W: Wie werden in der Medizin gute Entscheidungen getroffen? Medizin-ethische Diskussion, Teil 2. Hausarzt Praxis 2009;3:22-24. 